\title{
Perception of speech modulation cues by 6-month-old infants
}

\author{
Cabrera Laurianne $^{1,2}$, Bertoncini Josiane ${ }^{1}$, and Lorenzi Christian ${ }^{1,2}$ \\ ${ }^{1}$ Laboratoire de Psychologie de la Perception \\ CNRS, Université Paris Descartes \\ 45 rue des saints Pères, 75006 Paris, France \\ ${ }^{2}$ Département d'Etudes Cognitives, Institut d'Etude de la Cognition \\ Ecole normale supérieure, Paris Sciences et Lettres \\ 29 rue d'Ulm, 75005 Paris, France
}




\begin{abstract}
Purpose: This study assessed the capacity of 6-month-old infants to discriminate a voicing contrast (/aba/-/apa/) on the basis of amplitude modulation cues (AM, the variations in amplitude over time within each frequency band) and frequency modulation cues (FM, the oscillations in instantaneous frequency close to the center frequency of the band).

Method: Several vocoded speech conditions were designed to: (i) degrade FM cues in 4 or 32 bands, or (ii) degrade AM in 32 bands. Infants were familiarized to the vocoded stimuli for a period of either 1 or $2 \mathrm{~min}$. Vocoded speech discrimination was assessed using the head-turn preference procedure.
\end{abstract}

Results: Infants discriminated /aba/ from /apa/ in each condition. However, familiarization time was found to influence strongly infants' responses (i.e., their preference for novel versus familiar stimuli).

Conclusions: Six-month-old infants do not require FM cues, and can use the slowest $(<16$ Hz) AM cues to discriminate voicing. Moreover, six-month-old infants can use AM cues extracted from only four broad frequency bands to discriminate voicing.

Key Words: Speech perception, Vocoder, Modulation cues, Normal-hearing infants 


\section{Introduction}

Most recent studies about speech perception in infants have focused on how they acquire the phonological properties of their native language in a variety of learning contexts (see Kuhl et al., 2008, for a review). The present study takes a different approach by exploring how infants process the acoustic information related to phonetic differences. More precisely, this study aims to assess to which extent infants rely on low-level (i.e., sensory) spectro-temporal modulation cues to discriminate a voicing contrast (see also Bertoncini, Nazzi, Cabrera, \& Lorenzi, 2011).

The auditory mechanisms underlying speech perception in adulthood have been thoroughly described. Speech signals are decomposed by the auditory filters in the cochlea into many narrow frequency bands with a passband equal to one "equivalent-rectangular

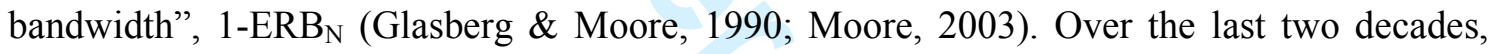
several psychophysical studies reconsidered speech-perception processes based on the general assumption that each $1-\mathrm{ERB}_{\mathrm{N}}$ wide band of speech should be viewed as a sinusoidal carrier with superimposed amplitude modulation (AM) and frequency modulation (FM) (e.g., Drullman, 1995; Shannon, Zeng, Kamath, Wygonski, \& Ekelid, 1995; Sheft, Ardoint, \& Lorenzi, 2008; Smith, Delgutte, \& Oxenham, 2002; Zeng et al., 2005). The AM component often referred to as the "acoustic temporal envelope" - corresponds to the relatively slow modulations in amplitude over time. The FM component - often referred to as the "acoustic temporal fine structure" - represents the relatively fast fluctuations in instantaneous frequency over time with average frequency close to the center frequency of the 1-ERB $\mathrm{N}_{\mathrm{N}}$ wide band.

Both AM and FM features are represented in the phase-locking pattern of auditorynerve fibers' discharges. For most adult mammals, the accuracy of neural phase locking to instantaneous frequency (and thus, FM cues) is constant up to about $1-2 \mathrm{kHz}$ and then 
declines so that phase locking is no longer detectable at about 5-6 kHz (e.g., Johnson, 1980; Kiang, Pfeiffer, \& Starr, 1965; Palmer \& Russell, 1986; Rose, Brugge, Anderson, \& Hind, 1967). In contrast, neural phase locking to AM cues remains accurate for carrier (audio) frequencies well beyond 6 kHz (e.g., Joris, Schreiner, \& Rees, 2004; Joris \& Yin, 1992; Kale \& Heinz, 2010). Moreover, several additional peripheral auditory mechanisms such as synaptic adaptation at the inner hair-cell level appear to limit AM coding beyond those that limit instantaneous-frequency coding (Joris \& Yin, 1992). These physiological data suggest some form of dissociation in the representation of AM and FM features in the early stages of the auditory system. However, it has been proposed that only the slowest FM $(<5-10 \mathrm{~Hz})$ cues are encoded in a purely temporal manner (that is, independently of AM) via phase locking in auditory-nerve fibers whereas faster FM cues $(>10 \mathrm{~Hz})$ are encoded via a place (i.e., tonotopic) mechanism (e.g.,Moore \& Sek, 1996; Saberi \& Hafter, 1995) ${ }^{1}$.

Phonetic features such as voicing, nasality, place and manner of articulation are signaled by various $\mathrm{AM}$ cues (e.g., the existence and duration of silent intervals are important in distinguishing voiced from voiceless plosives in intervocalic position; Rosen, 1992), which are relatively widespread or located in the high audio-frequency range (Rosen, 1992). Segmental cues to voicing and nasality are restricted to the low-mid audio frequency range (e.g., voiced sounds have a power spectrum heavily weighted to audio frequencies below 1 $\mathrm{kHz}$; Rosen, 1992) and are well represented in the pattern of phase locking in auditory-nerve fibers (e.g., Deng \& Geisler, 1987; Sinex \& Geisler, 1983), suggesting that voicing and nasality may also be signaled by FM cues encoded in a purely temporal manner.

\footnotetext{
${ }^{1}$ In this case, the differential attenuation of cochlear filtering converts the frequency excursions of FM into AM fluctuations at the output of auditory filters in the cochlea, a process sometimes referred to as "temporal envelope reconstruction" (see Gilbert \& Lorenzi, 2006; Zeng et al., 2004, for applications to speech perception).
} 
A number of speech perception studies have attempted to assess the relative importance of $\mathrm{AM}$ and FM cues in speech identification and phonetic-feature perception for normalhearing adults. These studies have used nonsense syllables, words or sentences processed by vocoders, that is signal-processing algorithms which extract and alter selectively AM and FM cues within specific analysis frequency bands (Dudley, 1939). After filtering out or scrambling the original AM or FM speech cues within each analysis frequency band, the resulting signal is assumed to retain mainly the AM or the FM speech cues (e.g., Gilbert \& Lorenzi, 2006; Shannon et al., 1995; Sheft et al., 2008; Smith et al., 2002; Zeng et al., 2005;). These studies indicated that normal-hearing adults can achieve high levels of speech intelligibility and phonetic-feature perception with speech stimuli vocoded to retain mainly the AM speech cues (stimuli referred to as "AM speech" thereafter). This was initially demonstrated by Shannon et al. (1995) who evaluated English-speaking adults' abilities to identify nonsense syllables in different speech-processing conditions. The AM-speech stimuli contained only AM cues extracted within a limited number of broad analysis frequency bands (1, 2, 3 or 4 bands) using a lowpass filter with a cutoff frequency varying from 16 to $500 \mathrm{~Hz}$. Syllable identification and phonetic-feature perception (i.e., voicing, manner and place of articulation) were poor for 1,2 , or 3 analysis frequency bands, but sharply increased with 4 bands, irrespective of the cutoff frequency of the lowpass filter used to extract AM. However, in order to reach such high levels of accuracy, participants required a relatively long training period ( 8 to 10 hours).

Other studies with vocoded signals revealed that adult listeners could also achieve high levels of speech perception with nonsense syllables or sentences while the physical signal retains mainly the FM cues (e.g., Gilbert \& Lorenzi, 2006; Hopkins, Moore, \& Stone, 2010; Lorenzi, Gilbert, Carn, Garnier, \& Moore, 2006; Sheft et al., 2008). However, participants 
required a much longer training period to identify accurately these "FM-speech" stimuli than their "AM-speech" counterparts. These data suggested that AM cues play a more important role than FM cues in accurate speech recognition. However, a number of studies conducted with adults tested with vocoded speech demonstrated that the relative importance of FM cues may increase when AM cues are degraded by various acoustic distortions (e.g., Ardoint \& Lorenzi, 2010; Gilbert, Bergeras, Voillery, \& Lorenzi, 2007; ; Gilbert \& Lorenzi, 2006; Hopkins, Moore, \& Stone, 2008; Nelson, Jin, Carney, \& Nelson, 2003; Qin \& Oxenham, 2003; Zeng et al., 2005). Indeed, speech perception was found to be poorer for "AM speech" than for "intact speech" (that is, for speech combining AM and FM cues within each frequency band) when stimuli were spectrally reduced or filtered, periodically interrupted or masked by interfering talkers or background noise (Eaves, Summerfield, \& Kitterick, 2011; Gnansia, Pean, Meyer, \& Lorenzi, 2009).

These data demonstrate that for adults, both AM and FM cues convey phonetic information whose relative weight may vary according to the listening conditions, and in a more general sense, as a function of speech redundancy. Thus, for adults, the loss of AM cues may be compensated for by relying more on FM cues. While numerous studies have investigated speech perception in adults, information is still lacking regarding the ability of neonates, infants and older children to use AM and FM cues in speech. Do infants use AM and FM cues in the same way as adults when listening to speech sounds? Do the acoustic degradations of AM and FM cues impair the performance of developing auditory mechanisms as much as that of mature (coupled) auditory and speech mechanisms? To our knowledge, only a few studies have directly addressed the developmental course of modulation perception using vocoded speech stimuli. 
In a pioneering study, Eisenberg, Shannon, Shaefer Martinez, Wygonski and Boothroyd (2000) assessed the ability of normal-hearing 7-and 10-year-old children and adults to identify nonsense syllables, words and sentences vocoded to retain only AM cues below $160 \mathrm{~Hz}$ within 4 to 8 analysis frequency bands (the FM carriers were replaced by noise in each band). The results showed that children less than 7 years required a higher frequency resolution (i.e., a greater number of analysis frequency bands) than 10 year-olds and adults to reach similar identification performance with the AM-speech stimuli. Interestingly, voicing perception (and especially, perception of the /sa/ versus /za/ contrast) was the poorest across all subjects groups.

This initial investigation was extended by Bertoncini, Serniclaes and Lorenzi (2009) to younger children aged between 5 and 7 years. A discrimination task was used with nonsense syllables vocoded to retain only AM cues below $64 \mathrm{~Hz}$ within 16 frequency bands (here, the FM carriers were replaced by pure tones with fixed frequencies). Bertoncini et al. (2009) found that normal-hearing 5-, 6-, and 7-year-old children were able to discriminate speech contrasts on the basis of AM cues at an adult level. This study also showed no significant difference in performance across age groups for voicing, place, manner, and nasality. Thus, the perception of AM cues below $64 \mathrm{~Hz}$ in speech appeared as robust for 5-year-old children as for adults when a discrimination task was used. However, consistent with the results of Eisenberg et al. (2000), children's discrimination scores were lowest for the voicing contrast. This finding was attributed to the attenuation of fundamental-frequency (F0) energy at the onset of voicing caused by the removal of FM cues.

Bertoncini et al. (2011) further explored the perception of voicing based on AM cues by testing six-month-old infants. A behavioral task (based on the head-turn preference procedure) was used to assess the ability of infants to discriminate pairs of AM-vocoded 
syllables such as /aba/ and /apa/. As in Bertoncini et al. (2009), the AM cues were extracted within 16 analysis frequency bands and lowpass filtered at $64 \mathrm{~Hz}$. Again, the original FM carriers were replaced by pure tones. The results showed that infants attended significantly longer to alternating sequences of /aba/ and /apa/ than to repeated sequences of either /aba/ or /apa/. This suggested that infants detected the alternation on the basis of the AM cues differentiating voicing information.

These studies revealed that infants and older children are able to discriminate phonetic features on the sole basis of AM cues. However, Eisenberg et al. (2000)'s study suggested that the capacity to resist degradations of AM and/or FM cues in speech sounds in a more demanding identification task may not be entirely mature before at least the age of 7 years. This is compatible with the outcome of several psychophysical studies conducted with nonlinguistic stimuli, showing that auditory sensitivity to AM and FM cues is not adult-like until around 10-11 years of age (e.g., Aslin, 1989; Colombo \& Horowitz, 1986; Hall \& Grose, 1994; Moore, Cowan, Riley, Edmondson-Jones, \& Ferguson, 2011; for a review, see also Saffran, Werker, \& Werner, 2006, and Werner \& Gray, 1998 ). Nonetheless, other studies conducted both in infants (e.g., Abdala \& Folsom, 1995; Levi, Folsom, \& Dobie, 1995; Olsho, 1985; Spetner \& Olsho, 1990) and in cats (Brugge, Javel, \& Kitzes, 1978; Kettner, Feng, \& Brugge, 1985) suggest that some aspects of frequency selectivity and neural phase locking in auditory-nerve fibers and brain-stem neurons should be mature from early in infancy. Regarding temporal resolution (that is the ability to follow changes in AM fluctuations as a function of time), the results vary according to the nature of the stimuli and according to the methods. In some studies, temporal resolution approaches an adult-like profile at about 6 months of age (e.g., Levi \& Werner, 1996; Trainor, Samuel, Desjardins, \& Sonnadara, 2001; Trehub, Schneider, \& Henderson, 1995). At the same time, other studies 
show differences across age groups, indicating that temporal sensitivity is generally poorer in infants and more dependent on the task or on sound complexity than in adults (Buss, Hall, Grose, \& Dev, 1999; Diedler, Pietz, Bast, \& Rupp, 2007; Smith, Trainor, \& Shore, 2006; Wightman, Allen, Dolan, Kistler, \& Jamieson, 1989). Nevertheless, it is generally admitted that, under some circumstances, the mechanisms governing auditory temporal resolution in infants operate qualitatively like those in adults (Werner, Marean, Halpin, Spetner, \& Gillenwater, 1992).

The above review of infants' abilities to process spectral and temporal auditory cues reveals important disparities between psychophysical studies using non-linguistic stimuli (e.g., pure tones, complex tones, noise bursts) and speech-acquisition studies using linguistic stimuli (e.g., syllables, words). The present study attempted to address these disparities by combining psychophysical and psycholinguistic methods to explore 6-month-old infants' abilities to process phonetic information on the basis of AM and FM speech cues. More precisely, the present study investigated whether or not 6-month-old infants discriminate a French voicing contrast (/aba/ versus /apa/) in spite of degradations of the AM and/or FM cues in speech sounds.

An impressive number of studies on the development of speech perception have shown that, during the first months of life, infants can discriminate many different phonetic contrasts, including non-native ones (e.g., Mattock, Molnar, Polka, \& Burnham, 2008; for a review see Kuhl, 2004, and Werker \& Tees, 1999). Voicing discrimination, and more precisely discrimination of the $/ \mathrm{b} /$ versus /p/ contrast, was demonstrated in infants as young as one month of age by Eimas, Siqueland, Jusczyk and Vigorito (1971) using the high-amplitude sucking method. However, at the end of the first year, speech perception is marked not only by a decline in non-native contrast discrimination (e.g., Werker \& Tees, 1983) but also by 
facilitation in native contrast discrimination (e.g., Kuhl et al., 2006). Moreover, a recent study indicated that between 4- and 8-months of age, French-learning infants become more sensitive to the French value $(0 \mathrm{~ms})$ of the voice-onset-time (VOT) boundary between voiced and voiceless plosive consonants (Hoonhorst et al., 2009). Therefore, the speech-processing mechanisms responsible for the perception of phonetic features such as voicing are developed at 6 months of age, but far from being entirely tuned to the typical contrastive patterns of the native language (e.g., Mattock et al., 2008; Werker \& Tees, 1983, but see Kuhl, Williams, Lacerda, Stevens, \& Lindblom, 1992).

The present study explored the early capacity of 6-month-old French-learning infants to use AM and FM cues in discriminating a voicing contrast. Two sets of syllables (/aba/ and /apa/ stimuli) were either left intact or processed by a multi-channel, noise-excited vocoder in order to: (i) degrade FM cues by replacing the FM carriers by bands of noise in each analysis frequency band, (ii) degrade AM cues - and especially F0-related periodic AM cues - by filtering out the AM components above $16 \mathrm{~Hz}$ in each frequency band, and (iii) degrade AM cues by reducing the frequency resolution of the vocoder (i.e., the number of analysis frequency bands) from $32,1-\mathrm{ERB}_{\mathrm{N}}$ wide to $4,8-\mathrm{ERB}_{\mathrm{N}}$ wide frequency bands. These three vocoded conditions were designed to assess whether normal-hearing 6-month-olds are able to discriminate a voicing contrast (/aba/ versus /apa/) on the sole basis of: (i) AM speech cues, (ii) the slowest $(<16 \mathrm{~Hz})$ AM speech cues, and (iii) the AM cues extracted from a limited number of broad frequency bands.

The intact and processed speech stimuli were presented to 6-month-old infants and an adaptation of the head turn preference procedure (HPP) was used to assess their discrimination ability. The procedure used in this experiment was adapted from the one introduced by Hirsh-Pasek et al. (1987). Our modified version of the HPP included a 
familiarization phase during which a stimulus is presented, followed by a test phase in which sequences of the (same) familiar stimulus alternated with sequences of a (different) novel stimulus. This version of HPP has been used recently in a study on melody discrimination in 2-month-old infants (Plantinga \& Trainor, 2009) and in several psycholinguistic studies assessing speech discrimination for 5-to-9-month-olds (e.g. Bosch \& Sebastian-Gallés, 2001; Höhle, Bijeljac-Babic, Herold, Weissenborn, \& Nazzi, 2009; Nazzi, Jusczyck, \& Johnson, 2000; Skoruppa et al., 2009). These studies showed that the familiarization period could induce in infants a preference for the familiar or novel sequences if and only if infants are able to discriminate these sequences ${ }^{2}$. In most studies, the duration of familiarization was set to 1 min but in some cases this duration was extended to 2 min (e.g. Bijeljac-Babic, Serres, Höhle, $\&$ Nazzi, 2012). In the present experiment, the duration of the familiarization phase was first set to $1 \mathrm{~min}$. For each experimental condition, we expected that the novel sequences would yield longer (correctly oriented) looking times than the familiar sequences.

\section{Experiment 1:}

\section{Participants}

Six-month-old infants were recruited from a database of birth announcements. All families were informed about the goals of the current study and provided a written consent before their participation, in accordance with current French ethical requirements.

Data from 88 infants from French-speaking families (22 infants per condition) were analysed in this experiment (40 boys and 48 girls; age range: 5 months 26 days; 7 months 10

\footnotetext{
${ }^{2}$ The procedure differs from a "spontaneous preference" paradigm in that the familiarization was achieved with sound A for half of the participants and with sound B for the other half, in such a way that preference for novel or for familiar stimuli cannot be confused with preference for one particular category (A or B). Second, it should be noted that while discrimination responses do not imply preference, displaying a preference necessitates discrimination between the two types of sequences.
} 
days; mean $=6$ months 16 days; $S D=9$ days). All infants are normal-hearing (based on parental report of newborn hearing screening results). The data from 37 additional infants were not included for the following reasons: fussing and crying $(n=22)$, looking time shorter than $1500 \mathrm{~ms}$ for one trial $(\mathrm{n}=6)$, outlier number of familiarization trials $(\mathrm{n}=6)$ and extreme mean looking times leading to outlier differences (more than, or less than the mean difference plus or minus $2 S D$, respectively) between novel and familiar series $(\mathrm{n}=3)$.

\section{Stimuli}

Speech signals were recorded in a soundproof room and digitized (16-bit resolution) at a 44.1-kHz sampling rate. A female French native speaker who was instructed to "speak clearly" produced sequences of /aba/ and /apa/. Sixteen tokens were selected from a large sample (around 150) in each phonetic category to be comparable in duration, intensity and pitch (in order to reduce variability across categories). Mean duration was $651.5 \mathrm{~ms}$ for /aba/ (range: 592-692 ms; $S D=36 \mathrm{~ms}$ ), and $630 \mathrm{~ms}$ for /apa/ (range: $570-702 \mathrm{~ms} ; S D=47 \mathrm{~ms}$ ). All stimuli were equated in global root-mean-square (RMS) level. The F0 was estimated at 242 Hz using the YIN algorithm (de Cheveigné \& Kawahara, 2002).

Four speech-processing conditions were used (the spectrograms of processed stimuli are shown in figure 1$)^{3}$. In the first condition (called "32-band AM+FM speech"), the original speech signal was decomposed into $32,1-\mathrm{ERB}_{\mathrm{N}}$-wide frequency bands using zero-phase, $6^{\text {th }}-$ order Butterworth bandpass filters (36 dB/octave rolloff) with central frequency (CF) ranging

\footnotetext{
${ }^{3}$ A pilot experiment using an $\mathrm{ABX}$, forced-choice discrimination task was initially conducted on 40 normalhearing adults using the intact and vocoded stimuli, in order to verify that the processed sounds can be discriminated. This pilot experiment showed that, after a very short practice, normal-hearing adults discriminated almost perfectly voiced and unvoiced consonants in each speech-processing condition ( $>90 \%$ correct discrimination). This finding is consistent with the outcome of the original speech-identification experiment conducted by Shannon et al. (1995), showing that voicing perception is nearly perfect in adults as long as AM cues below $16 \mathrm{~Hz}$ are presented in at least four broad spectral regions. Interestingly, all listeners reported having recognized the disyllables /aba/ and /apa/ except those presented with "4-band AM speech". In this speechprocessing condition, stimuli were not recognized as speech sounds.
} 
from 80 to $8,020 \mathrm{~Hz}$. The Hilbert transform was then applied to each bandpass filtered speech signal to extract the AM component and FM carrier. The AM component was low-pass filtered using a zero-phase Butterworth filter (36 dB/octave rolloff) with a cutoff frequency set to $\mathrm{ERB}_{\mathrm{N}} / 2$. The final narrow-band speech signal was obtained by multiplying each sample of the FM carrier by the filtered AM function. The narrow-band speech signals were finally added up and the level of the wideband speech signal was adjusted to have the same RMS value as the input signal. Thus, the vocoded speech signals retained the original AM and FM speech cues within each of the 32 analysis frequency bands.

In the second condition (called "32-band AM speech"), the same signal processing scheme was used as in the "32-band AM+FM speech" condition, except that the FM carrier was replaced by a band of pink noise in each analysis frequency band. Thus, the resulting vocoded speech signal retained AM speech cues within 32 bands, but discarded the original (within-channel) FM speech cues.

In the third condition (called "32-band $\mathrm{AM}<16 \mathrm{~Hz}$ speech"), the same signal processing scheme was used as in the "32-band AM speech" condition, except that the AM component was low-pass filtered with a cutoff frequency of $16 \mathrm{~Hz}$ for each of the 32 bands in order to remove fast, F0-related AM cues. Thus, the resulting vocoded speech signal retained mainly the slowest $(<16 \mathrm{~Hz})$ AM speech cues within 32 bands, and discarded the original FM speech cues.

In the last condition (called "4-band AM speech"), the same signal processing scheme was used as in the "32-band AM speech" condition, except that AM cues were extracted from only 4 , broad $\left(8-\mathrm{ERB}_{\mathrm{N}}\right.$ wide) frequency bands. Thus, the original FM speech cues were discarded, and AM cues were distorted substantially compared to the original AM speech 
cues. This vocoder also reproduces the sound processing typically achieved by current cochlear implant' sound processors (cf. Shannon et al., 1995).

/Insert Figure 1 about here/

In each condition, 4 different sequences were created. Each sequence was composed of 4 tokens of the same phonetic category, repeated 4 times in a different random order. Thus, all sequences have the same number of stimuli. Two sequences were used for the familiarization phase. Two sequences were used for the test phase. The tokens used in the test phase for each phonetic category were different from the ones used in the familiarization phase. The interstimulus interval was varied randomly between 390 and $600 \mathrm{~ms}$ all along the 16-item sequences. This random variation was introduced to prevent infants from using small variations in duration between items within and between categories. Finally, all the sound sequences were equated in duration $(18 \mathrm{~s})$.

\section{Procedure}

The experiment was conducted inside a sound-attenuated room where three lamps were fixed: a green one on the center wall, and a red one on each side wall. Below the green lamp was a hole for the lens of a video-camera. Out of the infant view, two loudspeakers were placed behind the red lamps and delivered the speech stimuli at $70 \mathrm{~dB}$ SPL (RMS) at the level of the infants' head. The infant was seated on the caregiver's lap in the center of booth. The caregiver was instructed not to speak or interfere in any way with the infant's behavior and wore ear plugs plus headphones delivering masking music.

The entire experimental session was controlled by a computer outside the booth, and a TV screen was connected to the camera. The experimenter sat outside the booth, looked at the video of the infant on the TV screen to monitor infant's looking behavior. The experimenter used a response box composed of three buttons corresponding to the three lights inside the 
booth. The response box was connected to the computer controlling the experiments. The experimenter pressed the buttons of the response box according to the direction of the infant's head (center, right or left). During the entire session, the experimenter wore headphones delivering masking music and was unaware of the nature of the displayed sequences (familiar or novel) during the test.

During the familiarization and the test phase, each experimental trial started by the blinking of the green center lamp. When the infant oriented to the green lamp, the experimenter pressed the "center" button, switching the green lamp off, and simultaneously one of the red lamps on. When the infant turned his/her head towards the red blinking lamp, the experimenter pressed the "right" or "left" button, triggering the presentation of the auditory stimulus from the corresponding loudspeaker. The trial was stopped when the infant turned his/her head away for more than $2 \mathrm{~s}$, corresponding to the button released by the experimenter, or when the end of the stimulus sequence was reached. Information about the duration of the head-turn was automatically stored on the computer.

During the familiarization phase, infants heard two sequences of the same phonetic category (half of the infants heard /aba/ and the other half /apa/). The familiarization trials continued (as described above) until infants listened to each sequence for a cumulative duration of $30 \mathrm{~s}$. This was achieved to complete $60 \mathrm{~s}$ of familiarization time. The number of trials necessary to reach this criterion differed across participants; those who reached the criterion with a number of trials significantly larger than the group mean were not included in the analyses.

In the test phase, two different sequences of the familiarized item, and two sequences of the novel item were displayed twice in random order and counterbalanced between the left and right side of presentation. Thus, the test phase was composed of 8 trials for each subject. 
The order of test sequences was counterbalanced between subjects (half of the subjects received a familiar stimulus sequence as first test trial while, the other half received a novel sequence as first test trial).

\section{Results}

Mean looking times were calculated for each participant across the 8 test trials ( 4 novel and 4 familiar sequences).

An analysis of variance (ANOVA) for repeated measures was run with 4 Conditions as the between-subject factor and 2 Types of sequences (Familiar versus Novel) as the withinsubject factor. This analysis did not indicate any main effect of Condition $(F(3,84)=0.78 ; p=$ $.51)$ or Type of sequence $(F(1,84)=0.26 ; p=.61)$, but a significant interaction between Condition and Type of Sequence $(F(3,84)=3.06 ; p=.033)$. This interaction is mainly due to the fact that in the "32-band AM+FM" condition, infants listened longer to the novel sequences than to the familiar sequences $(9.3 \mathrm{~s} ; S D=2.7 \mathrm{~s}$ versus $8.2 \mathrm{~s} ; S D=3.2 \mathrm{~s}$, respectively) compared to the "4-band AM" condition in which infants listened less to the novel sequences than to the familiar $(7.1 \mathrm{~s} ; S D=2.6 \mathrm{~s}$ versus $8.1 \mathrm{~s} ; S D=2.1 \mathrm{~s}$, respectively). In the other two conditions ("32-band AM" and "32-band AM<16 Hz"), the mean looking times were similar for the novel and the familiar sequences (see figure 2).

Planned comparisons confirmed that the significant interaction mentioned above is mainly due to the interaction between the "32-band AM+FM" and "4-band AM" conditions and Type of sequences $(F(1,84)=8.59 ; p=.004)$. Additionally, paired $t$-tests were carried out for each condition separately. A significant preference for the novel sequences was observed in the "32-band AM+FM" condition $(t(21)=2.33, p=.03)$, and a significant preference for the familiar sequences was observed in the "4-band AM" condition $(t(21)=2.43, p=.02)$. No 
significant difference was observed in the other two "32-band AM" and "32-band AM<16 Hz" conditions ( $p>.05$ in both conditions).

/Insert Figure 2 about here/

\section{Discussion}

As expected, infants exhibited significantly longer looking times for the novel stimuli in the "32-band AM+FM" speech condition, indicating that they discriminated /aba/ from /apa/ when provided with intact AM and FM speech cues. This novelty preference is a classical demonstration of infants' discrimination capacities. However, the familiarity preference can also be taken as an indication of discrimination (e.g., Hunter \& Ames, 1988). In behavioral procedures including a familiarization phase, familiarization is supposed to bias stimulus preference in such a way that all significant differences (whatever the direction, since familiarized stimuli were counterbalanced across subjects) would indicate that infants have processed novel and familiar stimuli differently. Here, an unexpected preference for familiar stimuli was observed for 6-month-old infants in the "4-band AM" speech condition, where the frequency resolution of the vocoder was reduced to 4 broad (i.e., 8-ERB $\mathrm{N}_{\mathrm{N}}$-wide) frequency bands. Although there are a number of hypotheses as to which factors affect the direction of preferences, no consensus has emerged (e.g., Hunter \& Ames, 1988; Rose, Gottfried, MelloyCarminar, \& Bridger, 1982; Thiessen \& Saffran, 2003; Wagner \& Sakovits, 1986). Hunter and Ames (1988) proposed a model of infant preferences for novel and familiar stimuli based on the interaction among three factors, namely age, familiarization time and task difficulty. According to this model, the present results in the "4-band AM" speech condition may be explained by the difficulty to process this relatively impoverished signal. In this speech condition, the original FM speech cues were discarded, and AM cues were distorted substantially compared to the "32-band AM+FM" condition and to the AM speech cues that 
listeners typically extract from the relatively narrow (1-ERB $\mathrm{N}_{\mathrm{N}}$-wide) frequency bands corresponding to the outputs of their cochlear filters (Kates, 2011).

The results obtained in the "32-band AM" condition were even more unexpected given that the stimuli used here were supposed to convey more accurate modulation information than the "16-band AM" speech stimuli previously used by Bertoncini et al. (2011). Indeed, in the present study, a higher frequency resolution was used and higher AM rates (e.g., periodic AM fluctuations at F0) were transmitted in high-CF analysis bands. Here, the AM component was lowpass filtered at $\mathrm{ERB}_{\mathrm{N}} / 2$ within each band, versus $64 \mathrm{~Hz}$ in Bertoncini et al. (2011). It is thus possible that the inherent random amplitude fluctuations of the noise carriers masked to some extent the original AM speech cues (e.g., Dau, Kollmeier, \& Kohlrausch, 1997a,b; Dau, Verhey, \& Kohlrausch, 1999; Lorenzi et al., 2001; see also Kates, 2011). Such a “modulation masking” effect did not occur in Bertoncini et al. (2011)' study because puretone carriers were used instead of band of noise. Alternatively, another factor could have accounted for this difference. As mentioned above, the preset duration of the familiarization period could have been inappropriate (i.e., too short) when artificially degraded stimuli such as noise-vocoded speech sounds were used. In Bertoncini et al.'s (2011), 6-month-old infants received no familiarization, and were found to prefer spontaneously alternating series of tonevocoded speech sounds to repeating ones. In the present version of the procedure, discrimination could require further perceptual processing than in the previous study, where the repetitive juxtaposition of /aba/ and /apa/ could have prompted immediate comparison. It may be the case that the information needed to represent a given stimulus category has to be gathered and correctly formatted before being compared to the (correctly formatted) other category. This is consistent with the studies conducted by Holt and Carney $(2005,2007)$ on children and adults. These authors proposed that "robustness" of the internal representation of 
speech stimuli and correct discrimination of a given speech contrast increase with increasing repetition/presentation of the stimuli. Again, the duration of the familiarization phase used in Experiment 1 might have been too short for infants to complete such a perceptual comparison, and to retrieve the original AM speech cues from the statistical amplitude fluctuations produced by noise carriers. Hunter and Ames (1988) indicated that the absence of any preference following a familiarization phase can be explained by a too short familiarization time. The duration necessary to be properly familiarized with a given stimulus may depend on the vocoded condition. The high variability in infants'responses observed in the "32-band $\mathrm{AM}$ " and "32-band $\mathrm{AM}<16 \mathrm{~Hz}$ " speech conditions may also question the effectiveness of the familiarization period, suggesting a transitional phase in preference in these 2 conditions (see also Hunter \& Ames, 1988; Rose et al., 1982).

The absence of a significant difference between the "32-band AM+FM", "32-band AM" and "32-band AM $<16 \mathrm{~Hz}$ " speech conditions precluded any conclusion about the effects of degrading AM and/or FM information. Thus, when the AM and FM speech cues are severely degraded, infants may need an extended exposure with the contrasted stimuli. In our view, this could also result in a more consistent effect of preference for one type of sequence. A second experiment in which the familiarization time was extended to a minimum of 2 min was conducted to test this hypothesis.

\section{Experiment 2:}

Four groups of twenty 6-month-old French-learning infants were tested with a minimum familiarization duration extended to $2 \mathrm{~min}$.

\section{Participants}


Data from 80 infants were analyzed (45 boys and 35 girls range: 5 months 23 days; 7 months 3 days; mean $=6$ months and 13 days; $S D=8$ days). The data from 18 additional infants were not included for the following reasons: fussing and crying $(\mathrm{n}=14)$, looking time lower than $1500 \mathrm{~ms}$ for one trial $(\mathrm{n}=1)$, outlier number of familiarization trials $(\mathrm{n}=2)$ and outlier looking time differences between novel and familiar conditions $(n=1)$.

\section{Stimuli}

Infants were presented with the same stimuli and sequences of stimuli as those used in Experiment 1 .

\section{Procedure}

The same procedure (HPP) was used, except that the familiarization time criterion was changed. In the familiarization phase, infants heard two sequences of the same phonetic category, and they had to listen to each sequence for at least $60 \mathrm{~s}$; therefore, the familiarization phase had a minimum cumulative duration of $120 \mathrm{~s}$. The test phase was left unchanged: each subject received 8 trials containing two different sequences of new tokens of the familiarized item, and two sequences of the novel item, that were played twice in a random order.

\section{Results}

As for Experiment 1, the mean looking times for novel and familiar stimuli were calculated for each participant on the 8 trials of the test phase. An ANOVA for repeated measures was run with 4 Conditions as the between-subject factor and 2 Types of sequences (Familiar versus Novel) as the within-subject factor. The main effect of Condition was not significant $(F(3,76)=1.29 ; p=.28)$. However, the analysis showed a significant effect of Type of sequence $(F(1,76)=8.88 ; p=.004)$ and a significant interaction between Condition 
and Type of sequence $(F(3,76)=5.67 ; p=.0015)$. Planned comparisons indicated that only the condition "32-band AM $<16 \mathrm{~Hz}$ " differed significantly from the three others $(\alpha=.05)$.

Once again, separate analyses revealed some differences between conditions. A significant preference for the novel sequences compared to the familiar ones was observed in three conditions: in the "32-band AM+FM" condition $(7.29 \mathrm{~s} ; S D=1.7 \mathrm{~s}$ versus $6.08 \mathrm{~s} ; S D=$ $2.07 \mathrm{~s}$, respectively; $t(19)=2.22, p=.039)$, in the "32-band AM" condition $(6.2 \mathrm{~s}, S D=2.6 \mathrm{~s}$ versus $4.8 \mathrm{~s}, S D=2.1 \mathrm{~s}$, respectively, $(t(19)=2.99, p=.014)$, and in the "4-band AM" condition ( $7.34 \mathrm{~s} ; S D=3.2 \mathrm{~s}$ versus $5.9 \mathrm{~s} ; S D=2.5 \mathrm{~s}$, respectively; $t(19)=2.50, p=.022)$. In the "32-band $\mathrm{AM}<16 \mathrm{~Hz}$ " condition, the mean looking time for novel sequences was shorter than for familiar sequences $(6.3 \mathrm{~s}, S D=3.1 \mathrm{~s}$ versus $7.3 \mathrm{~s}, S D=3.1 \mathrm{~s})$. This difference was significant $(t(19)=-2.55 ; p=.028)$, indicating that infants listened to familiar stimuli for a longer time in this condition (see figure 3).

/Insert Figure 3 about here/

\section{Discussion}

In this second experiment, each condition was re-tested with a preset familiarization time of $2 \mathrm{~min}$ instead of $1 \mathrm{~min}$ (as used in Experiment 1). With a longer familiarization time, the 6-month-old infants showed the classical pattern of preference for novelty in three conditions out of four. However, infants showed a preference for familiar stimuli in the "32band $\mathrm{AM}<16 \mathrm{~Hz}$ " condition, that is when the fastest ( $>16 \mathrm{~Hz}$ ) AM fluctuations related to F0 variations were reduced.

The results of this second experiment revealed that discrimination of /aba/ and /apa/ was possible, and indicated by a classical novelty preference in both "32-band AM" and "4band AM" conditions. The preference pattern in the "32-band AM $<16 \mathrm{~Hz}$ " condition turns out 
to be significant, and confirm the hypothesis that extended familiarization is necessary to evidence discrimination responses in some vocoder conditions.

\section{General discussion}

The present study was designed to explore how 6-month-old infants process a French voicing contrast on the basis of AM and FM cues, at an age when their perceptual mechanisms are not completely tuned to their native language. The disyllables (/aba/ versus /apa/) were used and several speech-processing conditions were designed to investigate whether infants discriminate this contrast when the modulation properties of speech sounds are severely degraded.

Discrimination of speech modulation cues. Six-month-old infants were found to discriminate the voicing contrast with the vocoded speech stimuli.

In the second experiment, results demonstrated that within-channel FM cues are not necessary to discriminate voicing at 6 months of age ( $c f$. "32-band AM" condition). They also demonstrated that infants could discriminate voicing in the absence of periodic AM fluctuations related to F0 ( $c f$. "32-band AM $<16 \mathrm{~Hz}$ " condition). However, even with an extended familiarization time, infants showed an opposite pattern of discrimination compared to the "32-band AM" condition where fast AM variations related to F0 were preserved. This suggests that infants are sensitive to a degradation of fast F0 cues in the AM domain, an indication that, as for adults (e.g., Rosen, 1992), the presence of fast, F0-related periodic information constitutes reliable cues to voicing cues for infants. This is also consistent with the outcome of previous psychophysical studies showing that the ability to detect changes in AM cues as a function of time - i.e., auditory temporal resolution - is efficient by 6 months of age (Levi \& Werner, 1996; for a review, see Saffran et al., 2006 and Werner \& Gray, 1998). 
In Experiments 1 and 2, the results showed that infants are able to discriminate speech signals containing only AM cues extracted within a small number of frequency bands ("4band AM" condition). Moreover, in Experiment 1, the preference pattern for familiar stimuli observed in this condition compared to the "32-band AM+FM" and "32-band AM" conditions suggests that infants are sensitive to a reduction in frequency resolution. This is consistent with the demonstration of adult-like frequency selectivity in infants by the age of 6 months (e.g., Abdala \& Folsom, 1995; Spetner \& Olsho, 1990).

The importance of auditory exposure to the stimuli. These findings suggest that at this early age, auditory processes are capable of making subtle speech distinctions despite severe distortions of speech modulation cues. Nevertheless, it is important to keep in mind that the demonstration of discrimination in infants was not straightforward in all conditions of degradation. In the "32-band AM+FM" condition, increasing the familiarization time did not affect the infants' pattern of responses. However, in the "4-band AM" condition, the increase in familiarization time by 1 min affected the infants' response pattern, which changed from a preference to familiar stimuli to a preference to novel stimuli. In the "32-band AM" condition, the extended auditory exposure led to a preference for the novel stimuli. In the "32-band AM< $16 \mathrm{~Hz}$ " condition, infants showed a significant preference for familiar sequences. Thus, a preset and relatively short time of exposure may not have allowed the 6-month-old infants to fully process speech cues in each vocoder condition, and build up the detailed representations of the vocoded signals required for robust discrimination ( $c f$. Holt, 2011).

This suggests that the present version of HPP based on a fixed familiarization time period might not have optimally assessed discrimination capacities of degraded stimuli (and quite unfamiliar speech sounds) in infants. The familiarization phase was aimed to provide an equivalent amount of auditory experience with a particular sound to a group of infants. 
However, it is important to keep in mind that vocoded stimuli strongly differed in terms of amount of spectro-temporal degradation. A procedure using an infant controlled habituation time would be more efficient to assess the discrimination capacity with such degraded speech stimuli. Future studies are warranted to assess whether or not familiarization time should be adapted to each infant in order to reduce inter-individual variability across the different conditions of degradation.

Different response patterns of infants. A non-classical preference pattern was observed for infants when AM and FM cues were simultaneously degraded as in the "32-band $<16 \mathrm{~Hz}$ " and "4-band AM" conditions (Experiment 2 and Experiment 1, respectively). In these two conditions, infants showed a preference for the familiar stimuli instead of the more typical preference for novel stimuli observed in the "32-band AM+FM" and "32-band AM" conditions. Altogether, these results are congruent with the assumption that a familiarity preference could be related to some difficulties in processing stimuli (Hunter \& Ames, 1988). This difference in response patterns suggests that the mechanisms involved in voicing perception may differ according to the severity of modulation distortions, and the subsequent "difficulty" in processing these highly distorted - and thus, unfamiliar - signals (Hunter \& Ames, 1988; Rose et al., 1982).

This suggestion is consistent with the fact that adult participants tested in a pilot study (see footnote 2) could not readily recognize the linguistic nature of the vocoded stimuli in the "4-band AM" condition, and required extensive training to identify consonants, vowels or sentences in the same speech-processing condition as previously shown by Shannon et al. (1995). This suggestion is also consistent with the notion that part of the processing mechanisms recruited for a given task may change whether adults are informed (or not) about the nature of what they are supposed to listen to (see Liebenthal, Binder, Piorkowski, \& 
Remez, 2003). When the nature of the stimuli is unknown and unfamiliar, perceptual mechanisms may differ from those activated by an intact speech signal.

In Experiment 2, the particular pattern of results observed in the "32-band AM< $16 \mathrm{~Hz}$ " condition may reveal the importance of the fast AM cues related to F0 fluctuations for voicing discrimination at 6 months.

Clinical implications. In the "4-band AM" condition, six-month-old infants (with normal hearing) were tested with speech stimuli processed by a vocoder simulating the sound processing typically achieved by current cochlear implant sound processors (e.g., Friesen, Shannon, Baskent, \& Wang, 2001; Shannon et al., 1995 ). The results showed that infants could still discriminate voicing despite important degradations in both AM and FM cues. However, a classical "novelty" discrimination response seems to require an extended exposure with the contrasted stimuli when the speech modulation cues were severely degraded by filtering or spectral reduction.

The implications of the present results for the understanding of speech perception capacities for infants wearing a cochlear implant should however be treated with caution. Indeed, the present results were obtained using degraded speech with normal-hearing infants, as well as a single speech contrast in a single context. Still, these results are very encouraging for understanding speech development in deaf infants wearing cochlear implants, and point to the necessity to investigate the basic auditory abilities needed to benefit from very early cochlear implantation (e.g., Holt \& Svirsky, 2008; Miyamoto, Houston, Kirk, Perdew, \& Svirsky, 2003; Nikolopoulos, Archbold, \& O’Donoghue, 1999; Svirsky, Robbins, Kirk, Pisoni, \& Myamoto, 2000). Further data will be needed to understand how the degraded signals delivered by cochlear implant processors are processed throughout the development of speech perception. In addition to their clinical relevance, these results might draw attention to 
the role of basic auditory processes upon early speech perception before or during the development of language-specific tuning (Kuhl et al., 2008).

\section{Conclusion}

The present study explored how infants process the acoustic information related to phonetic differences using a novel approach stressing the importance of modulation speech cues. The results indicate that the relatively fast AM and FM cues in speech are not necessary for French-learning 6-month-old infants to discriminate a voicing contrast in silence. These results demonstrate that the perception of voicing is robust as early as 6 months of age. However, the duration of initial auditory exposure (i.e., familiarization time) to the vocoded stimuli had to be increased to evidence discrimination abilities in all speech-processing conditions. Finally, the results show that normal-hearing infants can use the impoverished speech modulation cues delivered by prosthetic devices such as cochlear implants to discriminate voicing, provided they are sufficiently exposed and familiarized to these degraded speech stimuli.

\section{Acknowledgments}

This work was supported by ANR and program “Investissement d'avenir”(ANR-11-IDEX0001-02-PSL; Labex “Institut d'Etude de la Cognition”). C. Lorenzi was supported by a grant (HEARFIN Project) from ANR. The authors would like to thank warmly the parents for their participation in this study. 


\section{Figures}
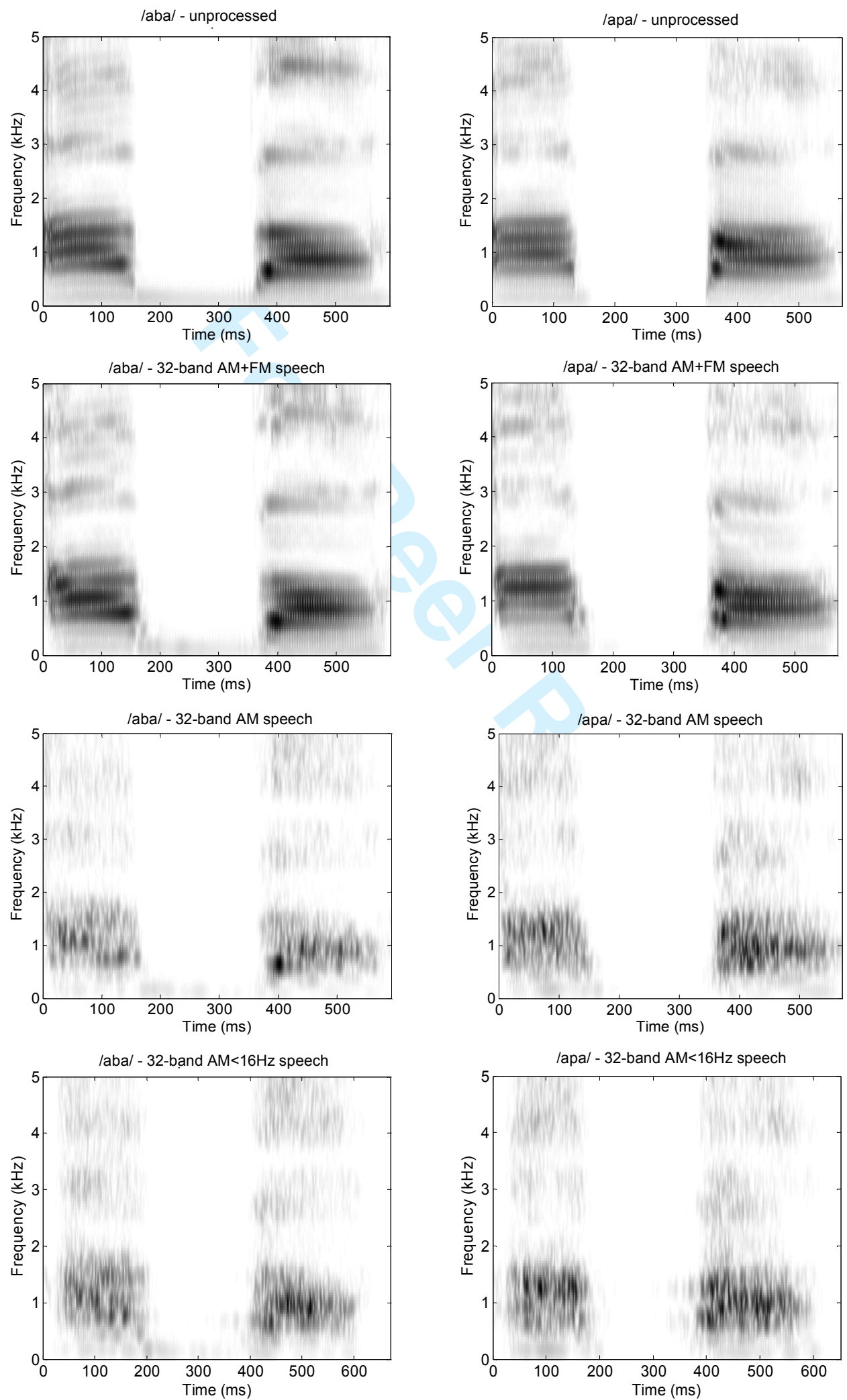

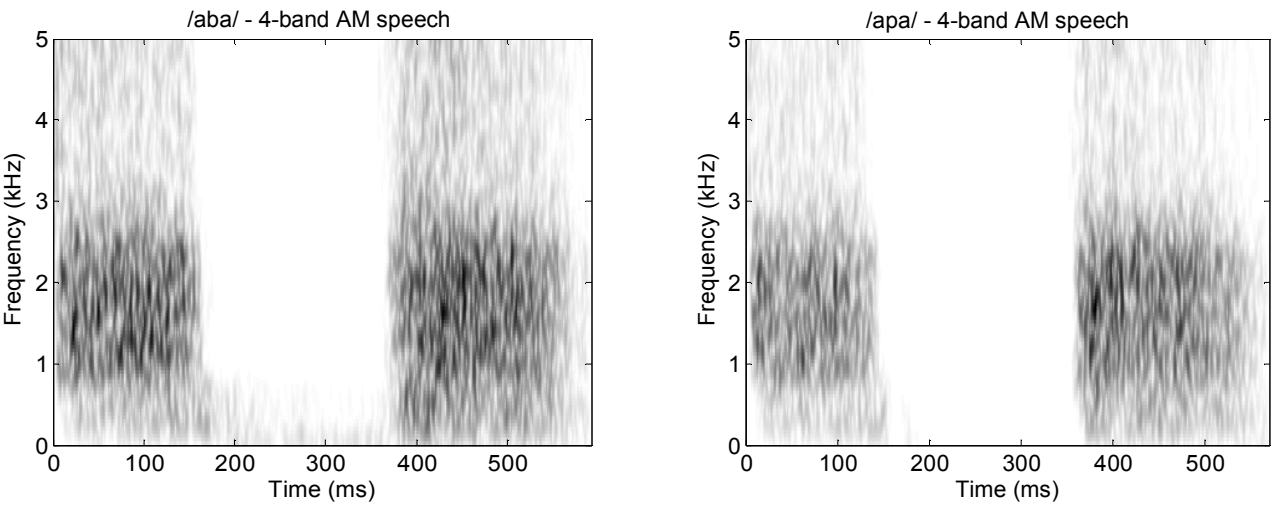

Figure 1 Spectograms of /aba/ (left panels) and lapa/ (right panels). From top to bottom panels: unprocessed, "32-band $A M+F M "$, "32-band $A M$ ", "32-band $A M<16 H z$ ", and "4band AM" speech conditions. 


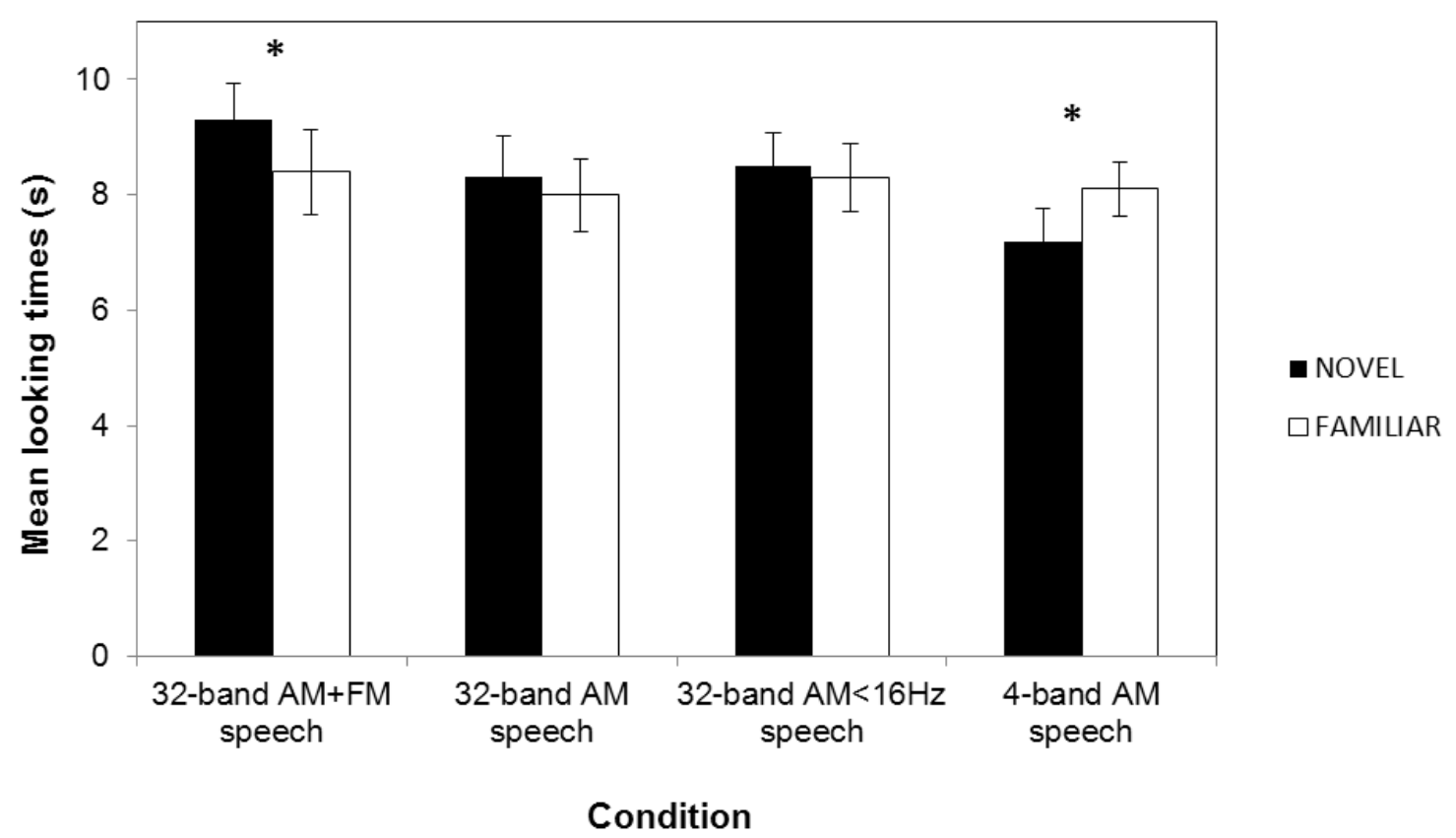

Figure 2 Mean looking times in Experiment 1 for familiar and novel stimuli during the test phase for each speech-processing condition (errors bars represent the standard errors). 


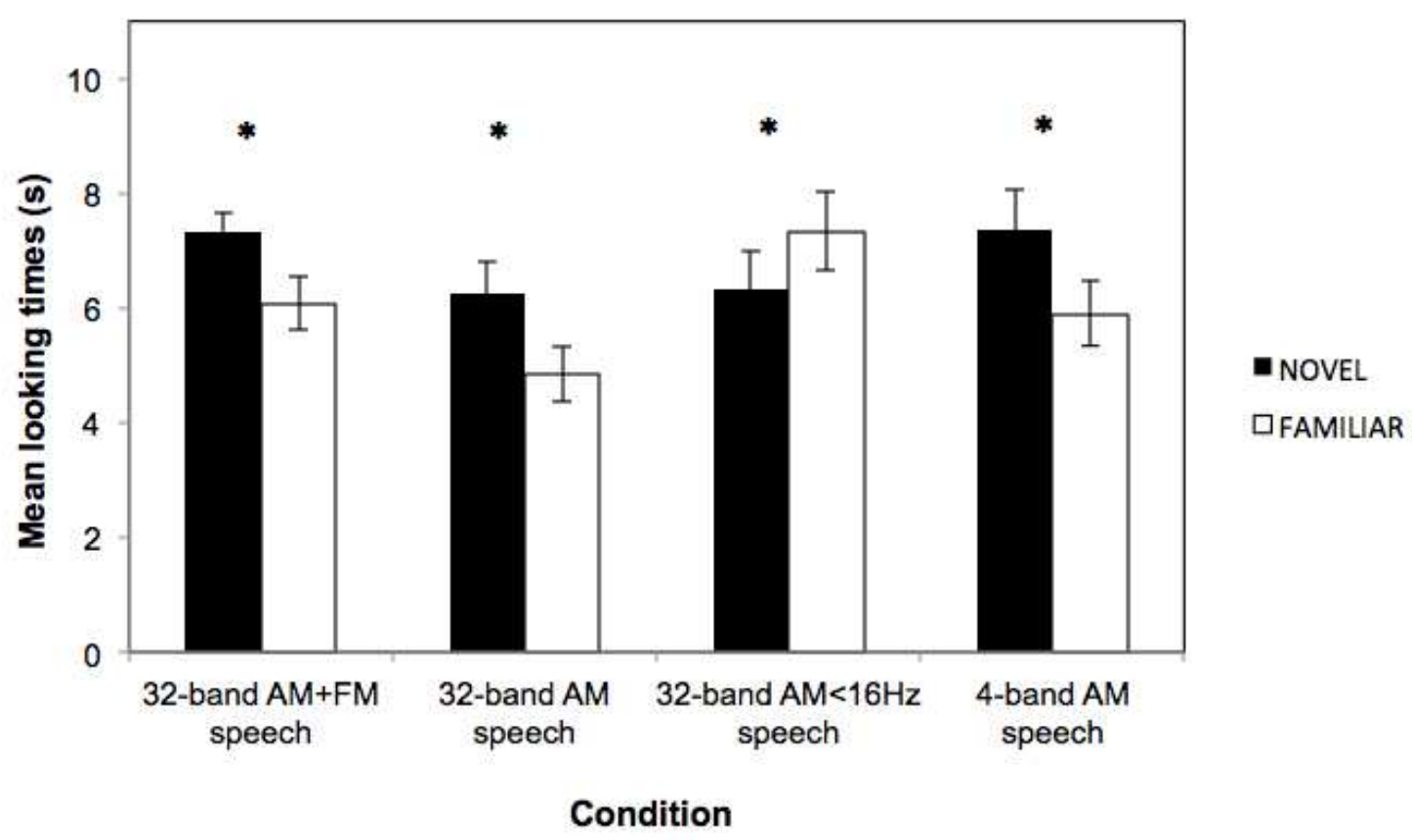

Figure 3 Mean looking times in Experiment 2 for familiar and novel stimuli during the test phase for each speech-processing condition (errors bars represent the standard errors). 


\section{References}

Abdala, C., \& Folsom, R.C. (1995). The development of frequency resolution in humans as revealed by the auditory brain-stem response recorded with notched-noise masking. Journal of the Acoustical Society of America, 98, 921-930.

Ardoint, M., \& Lorenzi, C. (2010). Effects of lowpass and highpass filtering on the intelligibility of speech based on temporal fine structure or envelope cues. Hearing research, 260, 89-95.

Aslin, R.N. (1989). Discrimination of frequency transitions by human infants. Journal of the Acoustical Society of America, 86, 582-590.

Bertoncini, J., Nazzi, T., Cabrera, L., \& Lorenzi, C. (2011). Six-month-old infants discriminate voicing on the basis of temporal envelope cues. The Journal of the Acoustical Society of America, 129, 2761-2764.

Bertoncini, J., Serniclaes, W., \& Lorenzi, C. (2009). Discrimination of speech sounds based upon temporal envelope versus fine structure cues in 5-to-7 year-old children. Journal of Speech Language and Hearing Research, 52, 682-695.

Bijeljac-Babic, R., Serres, J., Hohle, B., \& Nazzi, T. (2012). Effect of bilingualism on lexical stress pattern discrimination in French-learning infants. PLOS ONE, 7(2), e30843.

Bosch, L., \& Sebastian-Galles, N. (2001). Evidence of early language discrimination abilities in infants from bilingual environments. Infancy, 2, 29-49.

Brugge, J.F., Javel, E., \& Kitzes, L.M. (1978). Signs of functional maturation of peripheral auditory system in discharge patterns of neurons in anteroventral cochlear nucleus of kitten. Journal of Neurophysiology, 41, 1557-1559.

Buss, E., Hall, J.W.III, Grose, J.H., \& Dev, M.B. (1999). Development of adult-like performance in backward, simultaneous, and forward masking. Journal of Speech Language and Hearing Research, 42, 844-849.

Colombo, J., \& Horowitz, F.D. (1986). Infants attentional responses to frequency modulated sweeps. Child Development, 57, 287-291.

Dau, T., Kollmeier, B., \& Kohlrausch, A. (1997a). Modeling auditory processing of amplitude modulation : I. Modulation detection and masking with narrow-band carriers. Journal of the Acoustical Society of America, 102, 2892-2905. 
Dau, T., Kollmeier, B., \& Kohlrausch, A. (1997b). Modeling auditory processing of amplitude modulation : II. Spectral and temporal integration in modulation detection. Journal of the Acoustical Society of America, 102, 2906-2919.

Dau, T., Verhey, J., \& Kohlrausch, A. (1999). Intrinsic envelope fluctuations and modulationdetection thresholds for narrow-band carriers. Journal of the Acoustical Society of America, 106, 2752-2760.

de Cheveigné, A., \& Kawahara, H. (2002). YIN, a fundamental frequency estimator for speech and music. Journal of the Acoustical Society of America, 111, 1917-1930.

Deng L., \& Geisler, C.D. (1987). Responses of auditory-nerve fibers to nasal consonantvowel syllables. Journal of the Acoustical Society of America, 82, 1977-1988.

Diedler, J., Pietz, J., Bast, T., \& Rupp (2007). Auditory temporal resolution in children assessed by magnetoencephalography. Neuroreport, 18, 1691-1695.

Drullman, R. (1995). Temporal envelope and fine structure cues for speech intelligibility. Journal of the Acoustical Society of America, 97, 585-592.

Dudley, H. (1939). Remaking speech. The Journal of the Acoustical Society of America, 11, 165-165.

Eaves, J.M., Summerfield, A.Q., \& Kitterick, P.T. (2011). Benefit of temporal fine structure to speech perception in noise measured with controlled temporal envelopes. Journal of the Acoustical Society of America, 130, 501-507.

Eimas, P.D., Siqueland, E.R., Jusczyk, P., \& Vigorito, J. (1971). Speech perception in infant. Science, 171, 303-306.

Eisenberg, L.S., Shannon, R.V., Schaefer Martinez, A., Wygonski, J., \& Boothroyd, A. (2000). Speech recognition with reduced spectral cues as a function of age. Journal of the Acoustical Society of America, 107, 2704-2710.

Friesen, L.M., Shannon, R.V., Baskent, D., \& Wang, X. (2001). Speech recognition in noise as a function of the number of spectral channels: Comparison of acoustic hearing and cochlear implants. Journal of the Acoustical Society of America, 110, 1150-1163.

Gilbert, G., Bergeras, I., Voillery, D., \& Lorenzi, C. (2007). Effects of periodic interruption on the intelligibility of speech based on temporal fine-structure or envelope cues. Journal of the Acoustical Society of America, 122, 1336-1339.

Gilbert, G., \& Lorenzi, C. (2006). The ability of listeners to use recovered envelope cues from speech fine structure. Journal of the Acoustical Society of America, 119, 2438-2444. 
Glasberg, B.R., \& Moore, B.C.J. (1990). Derivation of auditory filter shapes from notchednoise data. Hearing Reseach, 47, 103-138.

Gnansia, D., Pean, V., Meyer, B., \& Lorenzi, C. (2009). Effects of spectral smearing and temporal fine structure degradation on speech masking release. Journal of the Acoustical Society of America, 125, 4023-4033.

Hall, J.W.III, \& Grose, J.H. (1994). Development of temporal resolution in children as measured by the temporal modulation transfer function. Journal of the Acoustical Society of America, 96, 150-154.

Hirsh-Pasek, K., Kemler Nelson, D.G., Jusczyk, P.W., Wright, K., Druss, B., \& Kennedy, L. (1987). Clauses are perceptual units for young infants. Cognition, 26, 269-286.

Höhle, B., Bijeljac-Babic, R., Herold, B., Weissenborn, J., \& Nazzi, T. (2009). The development of language specific prosodic preferences during the first half year of life: evidence from German and French. Infant Behavior and Development, 32, 262274.

Holt, R.F. (2011). Enhancing speech discrimination through stimulus repetition. Journal of Speech, Language, and Hearing Research, 54, 1431-1447.

Holt, R.F., \& Carney, A.E. (2005). Multiple looks in speech sound discrimination in adults. Journal of Speech, Language, and Hearing Research, 48, 922-943.

Holt, R.F., \& Carney, A.E. (2007). Developmental effects of multiple looks in speech sound discrimination. Journal of Speech, Language, and Hearing Research, 50, 1404-1424.

Holt, R.F., \& Svirsky, M.A. (2008). An exploratory look at paediatric cochlear implantation: Is earliest always best? Ear and Hearing, 29, 492-511.

Hopkins, K., Moore, B.C.J., \& Stone, M.A. (2008). Effects of moderate cochlear hearing loss on the ability to benefit from temporal fine structure information in speech. Journal of the Acoustical Society of America, 123, 1140-1153.

Hopkins, K., Moore, B.C.J., \& Stone, M.A. (2010). The effects of the addition of low-level, low-noise noise on the intelligibility of sentences processed to remove temporal envelope information. Journal of the Acoustical Society of America, 128, 2150-2161.

Hoonhorst, I., Colin, C., Markessis, E., Radeau, M., Deltenre, P., \& Serniclaes, W. (2009). French native speakers in the making: from language-general to language-specific voicing boundaries. Journal of Experimental Child Psychology, 104, 353-366. 
Hunter, M.A., \& Ames, E.W. (1988). A multifactor model of infant preferences for novel and familiar stimuli. Advances in infancy research, 5, 69-95.

Johnson, D.H. (1980). The relationship between spike rate and synchrony in responses of auditory-nerve fibers to single tones. Journal of the Acoustical Society of America, 68, 1115-1122.

Joris, P.X., \& Yin, T.C. (1992). Responses to amplitude-modulated tones in the auditory nerve of the cat. Journal of the Acoustical Society of America, 91, 215-232.

Joris, P.X., Schreiner, C.E., \& Rees, A. (2004). Neural processing of amplitude-modulated sounds. Physiological Reviews, 84, 541-577.

Kale, S., \& Heinz, M.G. (2010). Envelope coding in auditory nerve fibers following noiseinduced hearing loss. Journal of the Association for Research in Otolaryngology, 11, 657-673.

Kates, J.M. (2011). Spectro-temporal envelope changes changes caused by temporal finestructure modification. Journal of the Acoustical Society of America, 129, 3981-3990.

Kiang, N.Y., Pfeiffer, R.R., Warr, W.B., \& Backus, A.S. (1965). Stimulus Coding in the Cochlear Nucleus. Annals of Otology, Rhinology and Laryngology, 74, 463-485.

Kettner, R.E., Feng, J.Z., \& Brugge, J.F. (1985). Postnatal development of the phase-locked response to low frequency tones of the auditory nerve fibers in the cat. Journal of Neuroscience, 5, 275-283.

Kuhl, P.K. (2004). Early language acquisition: cracking the speech code. Nature Reviews. Neuroscience, 5, 831-43.

Kuhl, P.K., Conboy, B.T., Coffey-Corina, S., Padden, S., Rivera-Gaxiola, M., \& Nelson, T. (2008). Phonetic learning as a pathway to language: new data and native language magnet theory expanded (NLM-e). Philosophical Transaction of the Royal Society of London B Biological Science, 363, 979-1000.

Kuhl P.K., Stevens, E., Hayashi, A., Deguchi, T., Kiritani, S., \& Iverson, P. (2006). Infants show a facilitation effect for native language phonetic perception between 6 and 12 months. Developmental Science, 9, F13-F21.

Kuhl, P.K., Williams, K.A., Lacerda, F., Stevens, K.N., \& Lindblom, B. (1992). Linguistic experience alters phonetic perception in infant by 6 months of age. Science, 255, 606608. 
Levi, E.C., Folsom, R.C., \& Dobie, R.A. (1995). Coherence analysis of envelope-following responses (EFRs) and frequency-following responses (FFRs) in infants and adults. Hearing Research, 89, 21-7.

Levi, E. C., \& Werner, L. A. (1996). Amplitude modulation detection in infancy: Update on 3-month-olds. Abstracts of the Association for Research in Otolaryngology, 19, 142.

Liebenthal, E., Binder, J.R., Piorkowski, R.L., \& Remez, R.E. (2003). Short-term reorganization of auditory analysis induced by phonetic experience. Journal of cognitive neuroscience, 15, 549-58.

Lorenzi, C., Gilbert, G., Carn, H., Garnier, S., \& Moore, B.C.J. (2006). Speech perception problems of the hearing impaired reflect inability to use temporal fine structure. Proceedings of the National Academy of Sciences USA, 103, 18866-18869.

Lorenzi, C., Simpson, M.I.G., Millman, R.E., Griffiths, T.D., Woods, W.P., Rees, A., \& Green, G.G.R. (2001). Second-order modulation detection thresholds for pure-tone and narrow-band noise carriers. Journal of the Acoustical Society of America, 110, 2470-2478.

Mattock, K., Molnar, M., Polka, L., \& Burnham, D. (2008). The developmental course of lexical tone perception in the first year of life. Cognition, 106, 1367-1381.

Miyamoto, R.T., Houston, D.M., Kirk, K.I., Perdew, A.E., \& Svirsky, M.A. (2003) Language Development in Deaf Infants Following Cochlear Implantation. Acta OtoLaryngologica, 123, 241-244.

Moore, B.C.J. (2003). An introduction to the psychology of hearing, 5th Ed. (Academic Press, San Diego).

Moore, B.C.J., \& Sek, A. (1996). Detection of frequency modulation at low modulation rates: Evidence for a mechanism based on phase locking. Journal of the Acoustical Society of America, 100, 2320-2331.

Moore, D.R., Cowan, J.A., Riley, A.; Edmondson-Jones, A.M., \& Ferguson, M.A. (2011). Development of Auditory Processing in 6- to 11-Yr-Old Children. Ear \& Hearing, 32, 269-285.

Nazzi, T., Jusczyck, P.W., \& Johnson, E.K. (2000). Language discrimination by Englishlearning 5-month-olds: Effects of rhythm and familiarity. Journal of Memory and Language, 43(1), 1-19. 
Nelson, P.B., Jin, S.H., Carney, A.E., \& Nelson, D.A. (2003). Understanding speech in modulated interference: cochlear implant users and normal-hearing listeners. Journal of the Acoustical Society of America, 113, 961-969.

Nikolopoulos, T.P., Archbold, S.M., \& O’Donoghue, G.M. (1999). The development of auditory perception in children following cochlear implantation. International Journal of Pediatric Otorhinolaryngology, 49, 189-191.

Olsho, L.W. (1985). Infant auditory perception: Tonal masking. Infant Behavior Development, 8, 371-384.

Palmer, A.R., \& Russel, I.J. (1986). Phase-locking in the cochlear nerve of the guinea-pig and its relation to the receptor potential of inner hair-cells. Hearing research, 24, 1-15.

Plantinga, J., \& Trainor, L.J. (2009). Melody recognition by two-month-old infants. Journal of the Acoustical Society of America, 125(2), EL58-EL62.

Qin, M.K., \& Oxenham, A.J. (2003). Effects of simulated cochlear-implant processing on speech reception in fluctuating maskers. Journal of the Acoustical Society of America, $114,446-54$.

Rose, J.E., Brugge, J.F., Anderson, D.J., \& Hind, J.E. (1967). Phase-locked response to lowfrequency tones in single auditory nerve fibers of the squirrel monkey. Journal of Neurophysiology, 30, 769-793.

Rose, S.A., Gottfried, A.W., Melloy-Carminar, P., \& Bridger, W.H. (1982). Familiarity and novelty preferences in infant recognition memory: Implications for information processing. Developmental Psychology, 18(5), 704-713.

Rosen, S. (1992). Temporal information in speech: acoustic, auditory and linguistic aspects. Philosophical Transaction: Biological Sciences, 336, 367-373.

Saberi, K., \& Hafter, E.R. (1995). A common neural code for frequency- and amplitudemodulated sounds. Nature, 374, 537-539.

Saffran, J., Werker, J., \& Werner, L.A. (2006). The infant's auditory world: Hearing, speech and the beginnings of language. In D. Kuhn \& R.S. Siegler (ed.) Handbook of Child Psychology, Vol. 2, Cognition, Perception and Language (6th edition) New York: Wiley (pp. 58-108).

Shannon, R.V., Zeng, F.G., Kamath, V., Wygonski, J., \& Zkelid, M. (1995). Speech recognition with primarily temporal cues. Science, 270, 303-304. 
Sheft, S., Ardoint, M., \& Lorenzi, C. (2008). Speech identification based on temporal fine structure cues. Journal of the Acoustical Society of America, 124, 562-575.

Sinex, D.G., \& Geisler, C.D. (1983). Responses of auditory-nerve fibers to consonant-vowel syllables. Journal of the Acoustical Society of America, 73, 602-615.

Skoruppa, K., Pons, F., Christophe, A., Bosch, L., Dupoux, E., et al. (2009) Language specific stress perception by nine-month-old French and Spanish infants. Developmental Science, 12, 914-919.

Smith, N.A., Trainor, L.J., \& Shore, D.I. (2006). The development of temporal resolution: between-channel gap detection in infant and adult. Journal of Speech, Language, and Hearing Research, 49, 1104-1113.

Smith, Z.M., Delgutte, B., \& Oxenham, A.J. (2002). Chimaeric sounds reveal dichotomies in auditory perception. Nature, 416, 87-90.

Spetner, N.B., \& Olsho, L.W. (1990). Auditory Frequency Resolution in Human Infancy. Child Development, 61, 632-652.

Svirsky, M.A., Robbins, A.M., Kirk, K.I., Pisoni, D.B., \& Myamoto, R.T. (2000). Language development in profoundly deaf children with cochlear implant. Psychological Science, 11, 153-158.

Thiessen, E.D., \& Saffran, J.R. (2003). When cues collide: use of stress and statistical cues to word boundaries by 7- to 9-month-old infants. Developmental Psychology, 39, 706716.

Trainor, J.L., Samuel, S.S., Desjardins, R.N., \& Sonnadara, R.R. (2001). Measuring temporal resolution in infants using mismatch negativity. Neuroreport, 12, 2443-2448.

Trehub, S.E., Schneider, B.A., \& Henderson, J.L. (1995). Gap detection in infants, children, and adults. Journal of Acoustical Society of America, 98, 2532-2541.

Wagner, S., \& Sakovits, J. (1986). A process analyses of infant visual and cross-modal recognition memory: Implications for an amodal code. In L. Lipsitt, \& C. RoveeCollier, Advances in infancy research, 4, 196-217.

Werker, J.F., \& Tees, R.C. (1999). Influences on infant speech processing: toward a new synthesis. Annual Review of Psychology, 50, 509-35.

Werker, J.F., \& Tees, R.C. (1983). Developmental changes across childhood in the perception of non-native speech sounds. Canadian Journal of Psychology, 37, 278-86. 
Werner, L.A., Marean, G.C., Halpin, C.F., Spetner, N.B., \& Gillenwater, J.M. (1992). Infant auditory temporal acuity: gap detection. Child Development, 63, 260-272.

Werner, L.A., \& Gray, L. (1998). Behavioral studies of hearing development. In E.W Rubel, A.N.Popper, and R.R. Fay (Eds.). Development of the auditory system. Vol. 5, Springer handbook of auditory research. New York: Springer-Verlag, 12-79.

Wightman, F., Allen, P., Dolan, T., Kistler, D., \& Jamieson, D. (1989). Temporal resolution in children. Child Development, 60, 611-624.

Zeng, F.G., Nie, K., Liu, S., Stickney, G., Del Rio, E., Kong, Y.Y., \& Chen, H. (2004). On the dichotomy in auditory perception between temporal envelope and fine structure cues. Journal of the Acoustical Society of America, 116, 1351-1354.

Zeng, F.G., Nie, K., Stickney, G.S., Kong, Y.Y., Vongphoe, M., Bhargave, A., Wei, C., \& Cao, K. (2005). Speech recognition with amplitude and frequency modulations. Proceedings of the National Academy of Sciences USA, 102, 2293-2298. 